\title{
Dynamical modelling, stochastic simulation and optimization in the context of damage tolerant design
}

\author{
Sergio Butkewitsch \\ EMBRAER: Empresa Brasileira de Aeronáutica S.A., Structural Engineering Division, Structural Analysis Section, \\ Avenida Brigadeiro Faria Lima, 2170, São José dos Campos, SP, 12227-901, Brazil \\ Tel.: +55 123927 6139; Fax: +55 1239276600 ext. 2651; E-mail: sergio.choze@embraer.com.br
}

\begin{abstract}
This paper addresses the situation in which some form of damage is induced by cyclic mechanical stresses yielded by the vibratory motion of a system whose dynamical behaviour is, in turn, affected by the evolution of the damage. It is assumed that both phenomena, vibration and damage propagation, can be modeled by means of time depended equations of motion whose coupled solution is sought. A brief discussion about the damage tolerant design philosophy for aircraft structures is presented at the introduction, emphasizing the importance of the accurate definition of inspection intervals and, for this sake, the need of a representative damage propagation model accounting for the actual loading environment in which a structure may operate. For the purpose of illustration, the finite element model of a cantilever beam is formulated, providing that the stiffness matrix can be updated as long as a crack of an assumed initial length spreads in a given location of the beam according to a proper propagation model. This way, it is possible to track how the mechanical vibration, through its varying amplitude stress field, activates and develops the fatigue failure mechanism. Conversely, it is also possible to address how the effect of the fatigue induced stiffness degradation influences the motion of the beam, closing the loop for the analysis of a coupled vibration-degradation dynamical phenomenon. In the possession of this working model, stochastic simulation of the beam behaviour is developed, aiming at the identification of the most influential parameters and at the characterization of the probability distributions of the relevant responses of interest. The knowledge of the parameters and responses allows for the formulation of optimization problems aiming at the improvement of the beam robustness with respect to the fatigue induced stiffness degradation. The overall results are presented and analyzed, conducting to the conclusions and outline of future investigation.
\end{abstract}

Keywords: Coupled vibration-degradation, damage tolerance, simulation, optimization

\section{Introduction}

Aircraft are suitable representatives of a class of machinery supposed to operate for long service lives, economically delivering high levels of performance and enduring all kinds of adverse environments. In such circumstances, safety becomes the paramount design driver and the safety assurance for aging aircraft a major concern since the onset of the design development.

For modern aircraft, this concern is addressed by a set of measures aimed at resulting in damage tolerant designs. This means that all effort is expended to avoid the failure of any component but, even if such an unlikely event occurs, it is not sufficient to trigger hazardous failures.

The achievement of damage tolerance is possible due to a multidisciplinary approach, involving, among several issues, the identification of damage sources (environmental deterioration, accidental and fatigue damage), their evaluation and the estimation of their effects (loads, stresses, detail design, analysis and testing).

The aspects of aircraft manufacturing (parts production, assembly and quality control) and operation are deeply and extensively considered in this framework. Regarded in the broad sense, operation encompasses, besides the normal 
usage, the in-service introduction of structural modifications and repairs, the control and prevention of corrosion, the monitoring of widespread cracking and the definition of a comprehensive structural maintenance program.

The highlights of the structural maintenance plan are the major structural checks (when the aircraft are withdrawn from regular service and largely disassembled to replace the structural components that have reached their predicted useful lives regardless of their apparent integrity) and the periodic inspections (usually performed during normal operation or short stops) aimed at detecting incipient damage. Future design directions indicate that on-line structural health monitoring devices shall become an improvement by collecting vital information even during flight.

In order to establish the correct inspection intervals for each structural segment, aircraft developers must evaluate, since the design phase, how degradation damage shall evolve over time. For this purpose, a set of supposed damage scenarios is defined and the time frame in which they assume pre-defined risk levels is estimated. The structural maintenance plan is then formulated as to include inspections before some kind of damage can offer a significant risk.

It becomes evident that the accuracy of the estimates for the proper inspection intervals largely depends on the quality of the tools available to model and appraise how a given damage scenario should evolve. This work intends to present a contribution for the estimation of damage evolution in components subject to vibratory motion produced by dynamic loading.

\section{Basic formulation of the coupled vibration-degradation problem}

Temporal variations of the stress field and/or environmental conditions can cause irreversible changes in the characteristics of structural/mechanical systems, with the potential of significantly affecting their performance. These changes, referred to as degrading (or deterioration) phenomena, are usually not taken into account in the analysis of vibratory systems as an isolated discipline. Such analysis concentrates on the characterization of the response under various excitations assuming that the systems properties are fixed [12].

On the other hand, formulations dedicated to damage (mainly crack) propagation usually do not focus on the time dependency inherent to the oscillatory stress fields that occur due to dynamic motion.

Most important, the time evolution of each of these phenomena can be affected by the other, so that there is a mutual coupling between them. This means that the vibration motion, by inducing a varying amplitude stress field, activates and develops the fatigue failure mechanism. Conversely, the fatigue induced stiffness degradation influences the motion of the beam, closing the loop of a coupled vibration-degradation dynamical phenomenon.

In general, the coupled response-degradation problem for vibratory systems can be formulated as in Eqs (1) to (3):

$$
\begin{aligned}
& x(t)=F(t, D(t)) \\
& Q[x(t), \dot{x}(t), D(t), \dot{D}(t)]=0 \\
& x\left(t_{0}\right)=x_{0}, D\left(t_{0}\right)=D_{0}
\end{aligned}
$$

The motion response $x$ depends on all forcing sources, including the time varying damage $D(t)$. The coupled vibration-degradation process $Q$ equilibrates the states of motion and damage, given their respective state variables $x(t), \dot{x}(t)$ and $D(t), \dot{D}(t)$, besides the initial states $x_{0}$ and $D_{0}$.

Each one of the processes referenced by Eqs (1) to (3) is briefly examined in Sections 3 (for vibratory motion) and 4 (for crack propagation). A working model for their coupling is then developed in Section 5 and the remainder of the paper is devoted to applications and analysis.

\section{Outline of vibration analysis for design engineering}

The analysis of mechanical vibrations for the purpose of design engineering can be performed considering three major steps: 


\subsection{Formulation of the equations of motion}

This task is accomplished by applying fundamental mechanical laws to describe the system being analyzed in terms of its state of equilibrium. In the Newtonian approach, Eqs (4) and (5) are prescribed so that the vector sum of all time dependent forces $\vec{F}(t)$ and moments $\vec{M}(t)$ equals zero:

$$
\begin{aligned}
& \sum_{i} \vec{F}(t)=0 \\
& \sum_{i} \vec{M}(t)=0
\end{aligned}
$$

for all of the $i$ coordinates necessary and sufficient to completely and uniquely describe the motion of the system (i.e., its degrees-of-freedom). These forces and moments can be proportional to the system accelerations (inertia terms), velocities (dissipative terms) or displacements (stiffness terms).

On the other hand, the exchange of kinetic $(\mathrm{T})$ and elastic $(\mathrm{U})$ energy in a dissipative system (subject to the energy dissipation law R) can be considered through the variational formulation expressed in Eq. (6), known as the Lagrange Equation:

$$
\frac{d}{d t}\left(\frac{\partial T}{\partial \dot{q}_{i}}\right)-\frac{\partial T}{\partial q_{i}}+\frac{\partial U}{\partial q_{i}}+\frac{\partial R}{\partial \dot{q}_{i}}=Q_{i}
$$

The partial derivatives are taken with respect to the generalized coordinates $q$ (linear and/or angular displacements) or their time derivatives $\dot{q}$ (i.e., velocities) associated to the i degrees-of freedom of the system. The total derivative $d(\cdot) / d t$ is taken with respect to time and the right hand term is the vector of external forces and/or moments, that is, the generalized forces.

Either of these approaches conducts to a system containing i differential equations of motion, represented in matrix form by Eq. (7):

$$
[M]\{\ddot{x}(t)\}+[C]\{\dot{x}(t)\}+[K]\{x(t)\}=\{f(t)\}
$$

where $[\mathrm{M}],[\mathrm{C}]$ and $[\mathrm{K}]$ represent the inertia, damping and stiffness matrices associated to the accelerations $(\ddot{x}(t))$, velocities $(\dot{x}(t))$ and displacements $(x(t))$ respectively. The movement is induced by the action of the time varying external forces expressed by means of the vector $\{f(t)\}$. In the context of this paper, an important part of these forces appears due to stiffness changes promoted by the spread of some degradation agent (i.e., fatigue induced cracks). Besides damping variation is also induced by the crack degradation, this aspect is not addressed in this work as for modelling issues. A simpler working model is more suitable for the stochastic procedures described in Sections 6 and 7 .

\subsection{Solution of the equations of motion}

Although analytical solutions are available for Eq. (7), their implementation is often impractical, and numerical methods are employed to obtain approximate solutions, with satisfactory accuracy from the engineering viewpoint. A complete and throughout discussion about such solution methods is provided by [3].

\subsection{Comparison of the system dynamics with the excitation}

Regardless of the method, the solution of the equations of motion ultimately reveals how the system shall behave in the presence of a given excitation. Thus, the kinetic field of the system (its displacement, velocities and accelerations) can be expressed as a function of time or its reciprocal: the frequency.

Indeed, the Frequency Response Function is widely used as an engineering tool for the purpose of vibration analysis. By means of its graphical representation, as in Fig. 1, it is possible to infer about the dynamic behaviour of the system considering a range of possible excitation frequencies.

It should be stressed that this inherent dynamic response may be continuously changed along time if a damage phenomenon occurs simultaneously to the vibratory movement. 


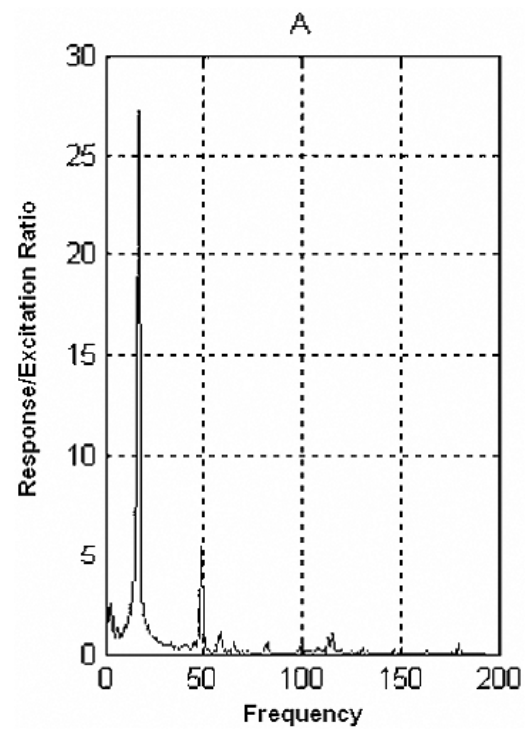

Fig. 1. Typical frequency response function for vibration analysis.

\section{Review of degradation (crack propagation) modelling}

The oscillatory motion associated with vibration phenomena results in time dependent displacement and, consequently, varying stress fields. Stresses that vary in magnitude and even signal (alternating between tension and compression) build up a scenario that is prone to the appearance of fatigue degradation. The stress variation can be accounted for by means of the stress ratio $\mathrm{R}$, that is, the quotient of the minimum by the maximum stress.

As to quantify this phenomenon along time, the basic quantity to be determined is the rate of change of the fatigue crack size (a) with time. In aircraft operation, time measurement for durability assessment is performed in terms of the number of load cycles or, in the context of this paper, vibration oscillations. Therefore, the dependence of crack size with respect to operating cycles $(\mathrm{N})$ can be expressed as shown in Eq. (8):

$$
\frac{d a}{d N}=f(\sigma, G, M)
$$

where the functional dependence is specified with respect to the stress field $(\sigma)$, the geometry $(\mathrm{G})$ and the material properties (M).

Several theories have been developed in order to establish a mathematical form to this law [4]. Equation (9) displays the so-called Paris law, which is indeed a set of equations, each one for a given value of the stress ratio R:

$$
\frac{d a}{d N}=c \cdot(\Delta K)^{m}
$$

The geometric shape correction factor is contemplated by the term c, while material properties are accounted for by the parameter $\mathrm{m}$. The stress intensity factor $\Delta \mathrm{K}$, given in Eq. (10), indicates the relation between the crack size and the variation in the stress intensity. The correction factor $\beta$ is determined empirically and the constant pi $(\pi)$ is approximately equal to 3.1416 .

$$
\Delta K=\beta \cdot(\Delta \sigma) \cdot \sqrt{\pi \cdot a}
$$

It is noticeable from Eqs (9) and (10) that the crack propagation phenomenon may become self-enhancing, since the crack growth rate depends on the stress intensity factor that, on its turn, depends on the crack size. Indeed, combinations of these key parameters lead to three distinct crack growth regimes, as shown in Fig. 2:

The value of the logarithmic relation between the crack growth rate with the number of loading cycles $(\mathrm{da} / \mathrm{dN})$ and the stress intensity factor $(\Delta \mathrm{K})$ defines which one is the prevailing regime. 


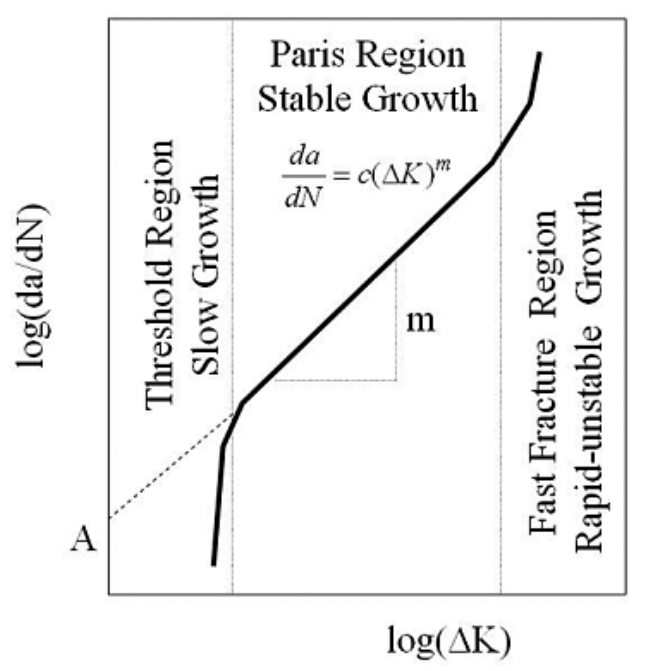

Fig. 2. Crack growth regimes, according to the Paris law.

Within the "Threshold Region", the crack growth is said to be slow because many load cycles occur with no actual growth. Then, the crack is increased of a size equivalent to one atomic spacing, followed by another series of load cycles without crack growth. Therefore, the crack growth (da), averaged along a series of loading cycles (dN), is lower than the material's atomic spacing. Operationally, it is customary to assume that this regime results in growth rates up to $10^{-8} \mathrm{~mm} / \mathrm{cycle}$. In terms of microstructure, this regime is characterized by the single shear between adjacent material layers, which is very sensitive to the stress ratio $(\mathrm{R})$ and the environmental agents (heat, humidity, etc.).

In the second regime, the Paris law can be applied based on the constant slope $\mathrm{m}$, the material characteristic whose identification demands experimental work. Typically, it is valued between 2 and 4 for metals and 4 and 100 for ceramics and thermo fix polymers, indicating more ductile and brittle behaviours, respectively. The governing parameter within this regime is the intensity factor $\Delta \mathrm{K}$, whilst the crack growth rate is rather insensitive with respect to the stress ratio $\mathrm{R}$.

Finally, there is a third regime in which the crack is said to be unstable, because the crack grows uncontrollably towards a complete failure, regardless of the environment effects, but highly influenced by the stress ratio $\mathrm{R}$ and the stress state itself.

From Fig. 2, it becomes evident that the design concern is geared towards the second regime, because:

a) There is no danger due to crack growth within the first regime.

b) There is nothing to be done since the third regime is reached.

Therefore, it is important to determine the crack size that marks the entrance into the second regime. In other words, a crack has to be detectable while going into this regime. Cracks can be detected using a variety of techniques, ranging from direct visual inspection to more sophisticated techniques, such as ultrasonics or X-rays. For analysis purposes, if no cracks are detectable, one must be conservative by assuming that a crack does exist, with an initial size just equivalent to the resolution of the available inspection system.

On the other hand, the critical crack size (the one from which the crack regime transitions from stable to unstable) has also to be determined. Therefore, a component may remain on service even if it has a detectable crack, given that its size is smaller than the critical one and that an inspection is provided before the critical size is reached. This operational mode represents the damage tolerant framework, which relies on the capacity of estimating the crack growth rates as to allow for the intercession of inspections, timely enough to avoid that the crack grows up to its critical size.

This work aims to contribute by formulating a model capable of performing the essential task of estimating the crack growth rate, accounting for the presence of dynamic loading. 


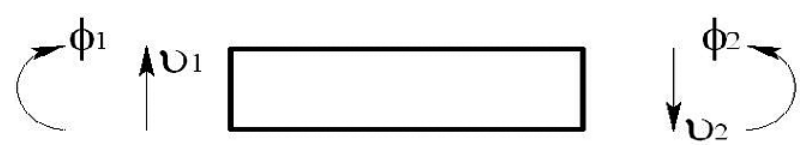

Fig. 3. General beam element subject to in-plane transversal loading.

\section{Formulation, results and performance of coupled vibration-degradation model}

\subsection{Formulation}

The dynamics of a cantilever beam is determined by means of the finite element method. The general beam element of length $\mathrm{L}$ subject to in-plane transversal loading is depicted in Fig. $3[1,8]$ :

Considering the translational $(\nu)$ and rotational $(\phi)$ degrees-of-freedom at both ends 1 and 2, and applying either Eqs (4) and (5) or Eq. (6), the stiffness and consistent mass matrices [8] assume the forms indicated by Eqs (11) and (12) respectively. The beam is assumed to be undamped.

$$
\begin{aligned}
& {[K]=\frac{E \cdot I}{L^{3}} \cdot\left[\begin{array}{llll}
12 & 6 \cdot L & -12 & 6 \cdot L \\
6 \cdot L 4 \cdot L^{2} & -6 \cdot L 2 \cdot L^{2} \\
-12-6 \cdot L & 12 & -6 \cdot L \\
6 \cdot L 2 \cdot L^{2} & -6 \cdot L & 4 \cdot L^{2}
\end{array}\right]} \\
& {[M]=\frac{\rho \cdot S \cdot L}{420} \cdot\left[\begin{array}{llll}
156 & 22 \cdot L & 54 & -13 \cdot L \\
22 \cdot L & 4 \cdot L^{2} & 13 \cdot L & -3 \cdot L^{2} \\
54 & 13 \cdot L & 156 & -22 \cdot L \\
-13 \cdot L-3 \cdot L^{2}-22 \cdot L & 4 \cdot L^{2}
\end{array}\right]}
\end{aligned}
$$

Each element is considered to be built of a material with modulus of elasticity $\mathrm{E}$ and the density $\rho$. The elemental cross section has area S and static inertia I. For the test case to be developed in this paper, three elements are used for the discretization of the cantilever beam, so that the crack is introduced at the element closest to the fixed end, where the bending stress is maximum (please refer to Fig. 5). The application of the proper boundary conditions and the assembly of the individual elemental matrices by means of the connecting degrees-of-freedom result in the complete stiffness and consistent mass matrices as in Eqs (13) and (14):

$$
\begin{aligned}
{[K]=\frac{E \cdot I}{L^{3}} \cdot\left[\begin{array}{llllll}
24 & 0 & -12 & 6 \cdot L & 0 & 0 \\
0 & 8 \cdot L^{2} & -6 \cdot L & 2 \cdot L^{2} & 0 & 0 \\
12 & -6 \cdot L & 24 & 0 & -12 & 6 \cdot L \\
6 \cdot L & 2 \cdot L^{2} & 0 & 8 \cdot L^{2} & -6 \cdot L 2 \cdot L^{2} \\
0 & 0 & 12 & -6 \cdot L & 12 & 6 \cdot L \\
0 & 0 & 6 \cdot L & 2 \cdot L^{2} & -6 \cdot L & 4 \cdot L^{2}
\end{array}\right] } \\
{[M]=\frac{\rho \cdot S \cdot L}{420} \cdot\left[\begin{array}{llllll}
312 & 0 & 54 & 13 \cdot L & 0 & 0 \\
0 & 8 \cdot L^{2} & 13 \cdot L-3 \cdot L^{2} & 0 & 0 \\
54 & 13 \cdot L & 312 & 0 & 54 & 13 \cdot L \\
-13 \cdot L & 3 \cdot L^{2} & 0 & 8 \cdot L^{2} & 13 \cdot L & 3 \cdot L^{2} \\
0 & 0 & 54 & 13 \cdot L & 156 & 22 \cdot L \\
0 & 0 & 13 \cdot L-3 \cdot L^{2}-22 \cdot L & 4 \cdot L^{2}
\end{array}\right] }
\end{aligned}
$$

Besides the finite element modelling of the vibration motion, a discrete version [2] of the Paris law (for $\mathrm{j}$ oscillations) is implemented as shown in Eq. (15), so that the time dependent vibratory motion and crack propagation can be coupled together, according to the scheme depicted in Fig. 4.

$$
\Delta a=\left\{c \cdot\left[\beta_{j} \cdot\left(\Delta \sigma_{j}\right) \cdot \sqrt{\pi \cdot a_{j}}\right]^{m}\right\} \cdot \Delta N_{j}
$$




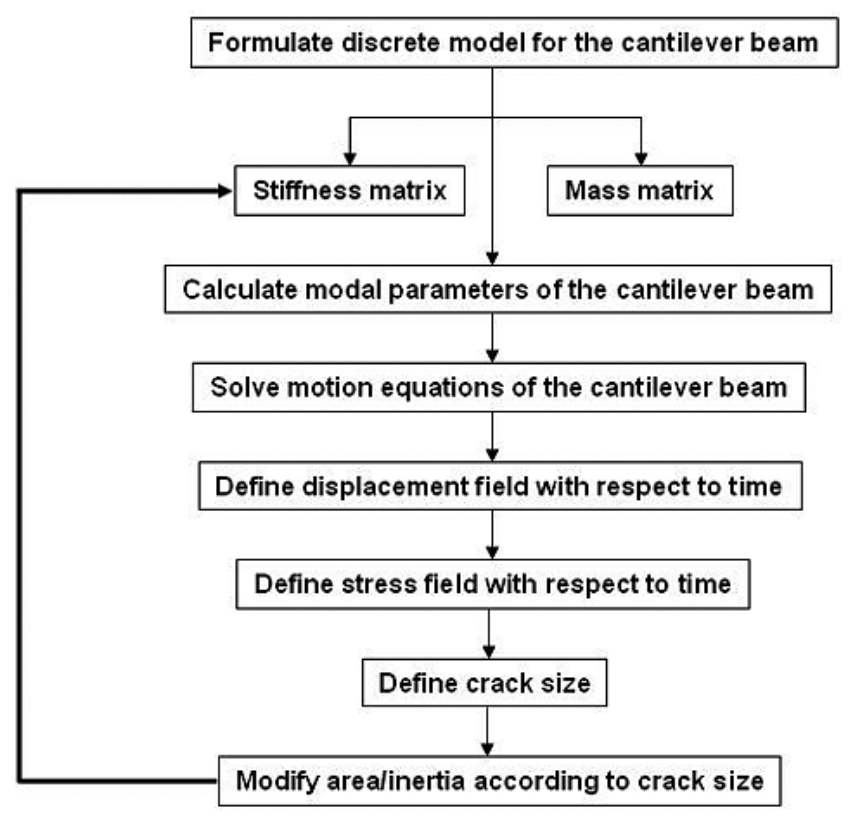

Fig. 4. Flowchart of numerical scheme to couple the vibration and degradation phenomena.

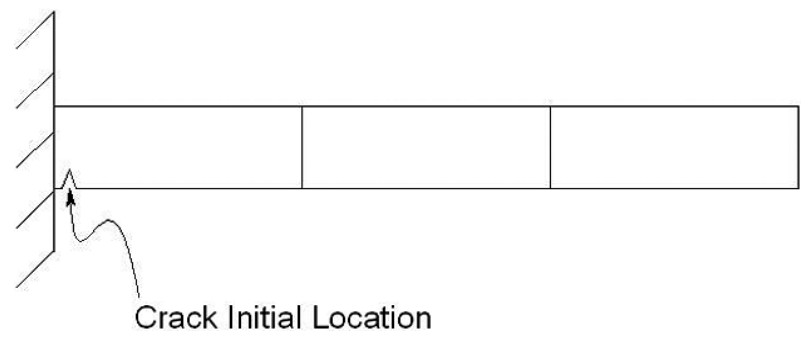

Fig. 5. Indication of crack position.

\subsection{Results and performance}

Initially, the working model described in section 5.1 is used considering a cantilever beam $0.30 \mathrm{~m}$ long, whose rectangular cross section measures $0.01 \mathrm{~m}$ and $0.02 \mathrm{~m}$ for the base $(\mathrm{b})$ and the height $(\mathrm{h})$ respectively. The material is aluminum, with elasticity modulus equal to $7.1020 \times 10^{10} \mathrm{~N} / \mathrm{m}^{2}$ and weight density equal to $28600.00 \mathrm{~N} / \mathrm{m}^{3}$. With these characteristics, the fundamental vibrating frequency of the beam is $11.21 \mathrm{~Hz}$ considering its intact state.

Under the action of a dynamic force with $5.00 \mathrm{~N}$ in magnitude, alternating at $5.00 \mathrm{~Hz}$ (about half of the fundamental frequency) and located at the beam tip, a crack of initial length equal to $0.50 \times 10^{-3} \mathrm{~m}$ is supposed to arise near to the clamped end (as schematically indicated in Fig. 5), since this is the region subject to the maximum bending moment. This flaw is accounted for by a reduction in the beam height, equivalent to the crack length at each time instant. Numerically, this is accounted for by updating the element cross section area at each crack growth step.

The graphs presented in Figs 6, 7 and 8 provide some insight about the effect of the growth of this crack over the system dynamics and vice-versa.

Figures 6 and 7 complement each other in the sense that the stiffness loss mechanism along the loading cycles is highlighted. While the crack grows, as shown in Fig. 6, the fundamental vibrating frequency of the beam decays, according to Fig. 7.

Both phenomena are highly non-linear with respect to the load cycles, especially when the crack reaches its critical size and becomes unstable. At this point, the stress level at the remaining (non-cracked) material is enough to promote a failure, which is preceded by abrupt changes both in crack growth and vibrating frequency decrease. 


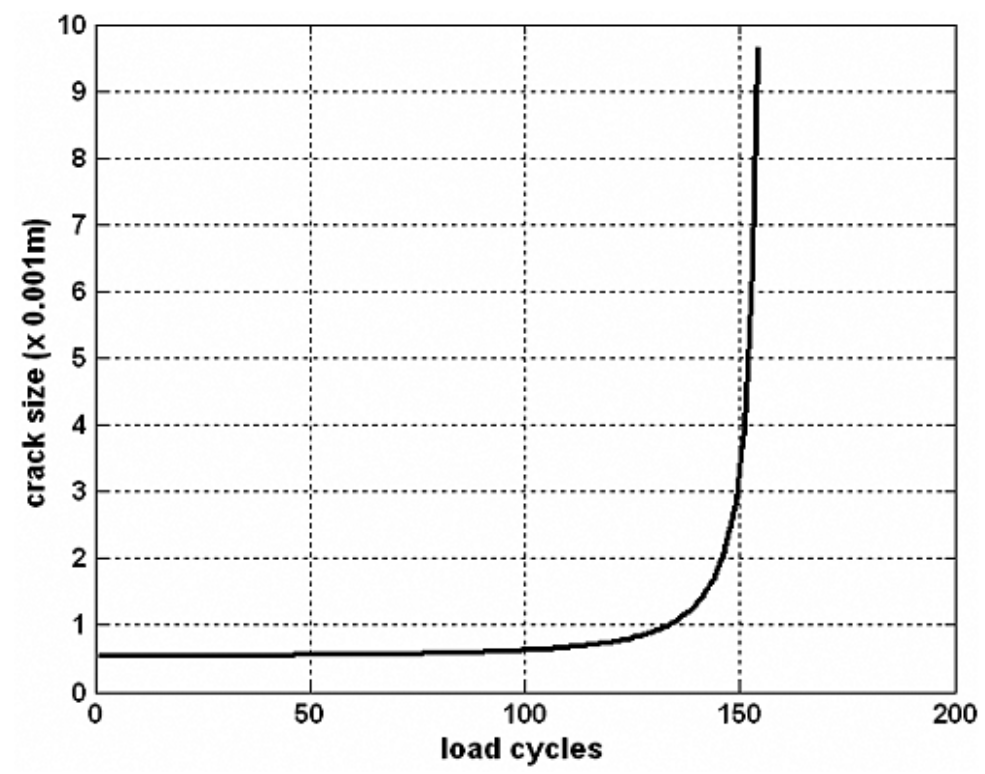

Fig. 6. Crack propagation along load cycles.

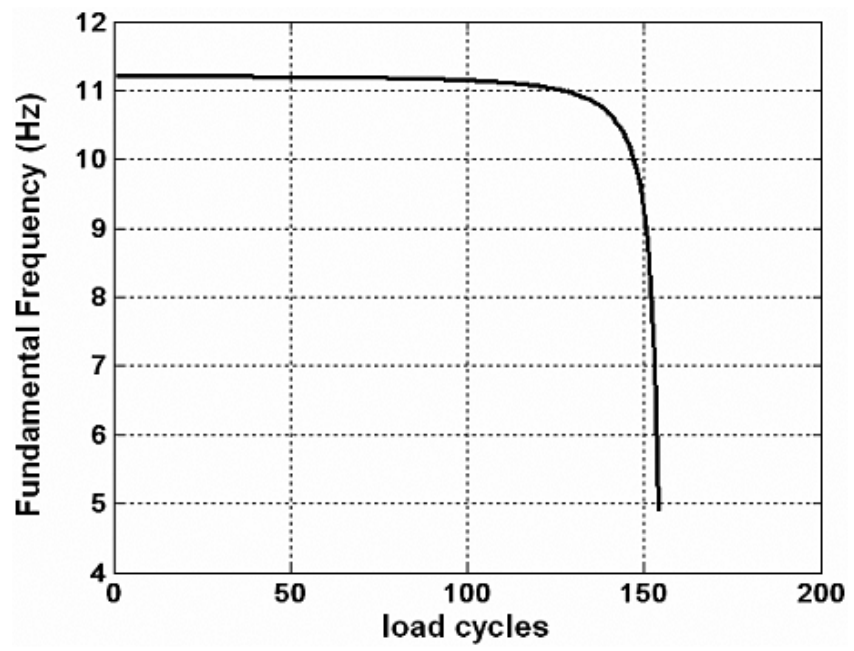

Fig. 7. Fundamental frequency decay with crack propagation.

Also some insight is provided about the proportions of the rates of change of the relevant physical quantities with the spread of the degradation. At about 150 load cycles, a sudden change occurs to both the crack size and the natural frequency values, heralding the failure. From the beginning of the oscillatory loading up to this point, the crack increases from $0.5 \times 10^{-3} \mathrm{~m}$ to $2.0 \times 10^{-3} \mathrm{~m}$, a $300.00 \%$ variation. In this same interim, the fundamental frequency drops from $11.21 \mathrm{~Hz}$ to $9.74 \mathrm{~Hz}$, representing a $13.11 \%$ change. Therefore, up to the crack critical size, the value of the fundamental vibrating frequency is much less sensitive to the degradation than the crack growth itself but, from the unstable crack size and on, the rates of change of these two quantities develop at the same (sudden) pace.

The important aspect contained in Fig. 8 is that these two symptoms of the degradation phenomenon (crack growth and frequency decay) can be linearly related to each other. This feature may be very convenient as to build analytical or empirical models for the study of the coupled vibration-degradation process. For example, one can measure the variation on the fundamental frequency and accurately relate it to estimates of crack sizes by means of a simple linear relationship. The frequency measurement usually poses less experimental difficulties and costs than 


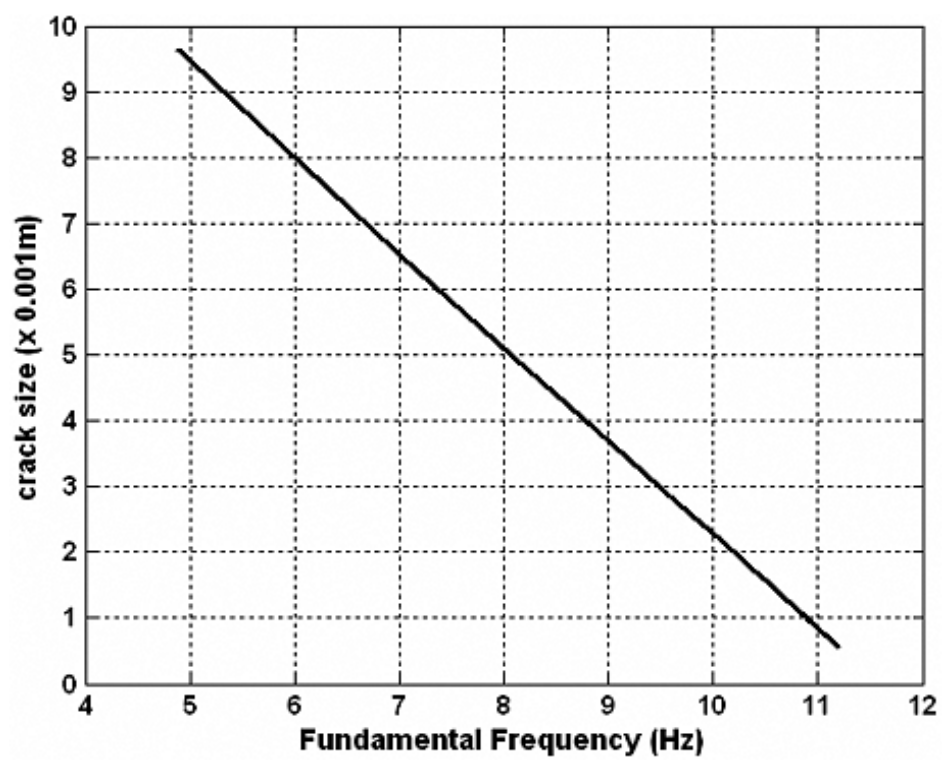

Fig. 8. Relation between fundamental frequency and crack size during degradation spread.

the direct evaluation of the crack size, mainly when there are access obstacles to the structural component of interest. However, as pointed out in the previous paragraph, the sensitivity of the natural frequency with respect to the damage accumulation is considerably lower than that of the crack growth rate itself, which results in practical difficulties to monitor the crack growth by means of the frequency decay, chiefly for large structural components presenting mild damage. Therefore, the main purpose of the working model developed herein is, as stated in Sections 1 and 2 , to improve life predictions when the actual loading acting over the structure is dynamic. Further applications are proposed in Sections 6 and 7.

\section{Stochastic simulation}

With the availability of a working model such as that described in Section 5, further types of engineering analysis can be developed. One shall consider, for instance, that in the construction of an aircraft there is a very stringent quality control effort, so that variations in material properties and physical dimensions are driven to a minimum and, when they occur, they are quantified and recorded. The central tendency and dispersion metrics contained in such databases can be used in order to estimate the effect of dimensional and material variations on the behaviour of a damaged cantilever beam subject to dynamic loading.

Assuming a conservative approach, the dispersion levels used in this work, measured in terms of standard deviations from the mean value, are significantly higher than the usual. Besides, they are fitted into a log-Normal probability distribution $[9,11]$, in which most of the observations fall below the average.

Another variability source refers to the loading environment itself. In this case, conservatism is achieved through a rationale opposed to that of the log-Normal distribution, that is, the majority of the values shall be concentrated above the average. For this purpose, a Beta probability distribution with the first shape parameter larger than the second one [5] is chosen. Figure 9 illustrates the shapes of the probability density functions assumed for each variability source.

Taking these aspects into account, a total of 20 runs is performed within a stochastic simulation with the simultaneous variation of material, cross section dimensions and load magnitude. Among the many results that can be obtained, this section focuses on the number of load cycles in which the failure occurs for each of the stochastic scenarios. Thus, a time-to-failure (TTF) data set is generated and a Weibull probability analysis [10] is performed as shown in Table 1: 
Table 1

Weibull analysis of the time-to-failure data from the stochastic simulation of the damaged vibrating beam

\begin{tabular}{ccccccc}
\hline $\begin{array}{c}\text { Stochastic } \\
\begin{array}{c}\text { Simulation } \\
\text { Run }\end{array}\end{array}$ & Time-to-Failure & $\begin{array}{c}\text { Ranked } \\
\text { Time-to-Failure }\end{array}$ & $\begin{array}{c}\text { Natural Logarithm } \\
\text { of Ranked } \\
\text { Time-to-Failure }(\mathrm{X})\end{array}$ & Rank & $\begin{array}{c}\text { Median Rank } \\
\text { Transformed } \\
\text { Median Rank } \\
(\text { Y) }\end{array}$ \\
\hline 1 & 72 & 60 & 4.094344562 & 1 & 0.034313725 & -3.354802509 \\
2 & 82 & 63 & 4.158883083 & 2 & 0.083333333 & -2.441716399 \\
3 & 72 & 72 & 4.219507705 & 3 & 0.132352941 & -1.952137671 \\
4 & 86 & 72 & 4.262679877 & 4 & 0.181372549 & -1.608807204 \\
5 & 78 & 73 & 4.33073334 & 5 & 0.230392157 & -1.339891087 \\
6 & 228 & 73 & 4.369447852 & 6 & 0.279411765 & -1.115695152 \\
7 & 82 & 75 & 4.430816799 & 7 & 0.328431373 & -0.920953918 \\
8 & 204 & 76 & 4.49980967 & 8 & 0.37745098 & -0.746689513 \\
9 & 342 & 77 & 4.564348191 & 9 & 0.426470588 & -0.587084006 \\
10 & 144 & 78 & 4.663439094 & 10 & 0.475490196 & -0.438053654 \\
11 & 242 & 82 & 4.744932128 & 11 & 0.524509804 & -0.296508894 \\
12 & 75 & 82 & 4.770684624 & 12 & 0.573529412 & -0.159920103 \\
13 & 73 & 86 & 4.820281566 & 13 & 0.62254902 & -0.026021058 \\
14 & 73 & 91 & 4.86753445 & 14 & 0.671568627 & 0.107442983 \\
15 & 176 & 144 & 4.9698133 & 15 & 0.720588235 & 0.24300008 \\
16 & 60 & 176 & 5.170483995 & 16 & 0.769607843 & 0.383882124 \\
17 & 77 & 204 & 5.318119994 & 17 & 0.818627451 & 0.534855821 \\
18 & 63 & 228 & 5.429345629 & 18 & 0.867647059 & 0.704227134 \\
19 & 91 & 242 & 5.488937726 & 19 & 0.916666667 & 0.910235093 \\
20 & 76 & 342 & 5.834810737 & 20 & 0.965686275 & 1.21556827 \\
\hline
\end{tabular}

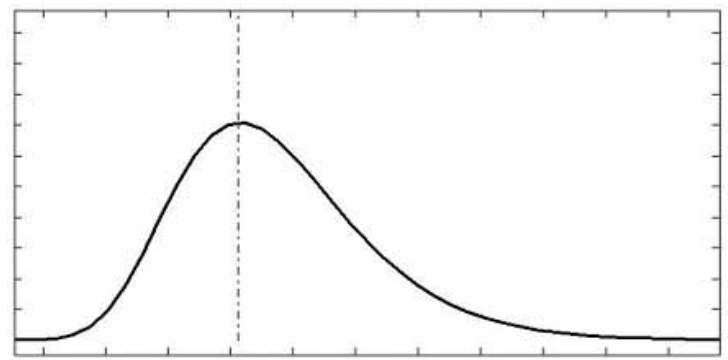

(a) Log-normal

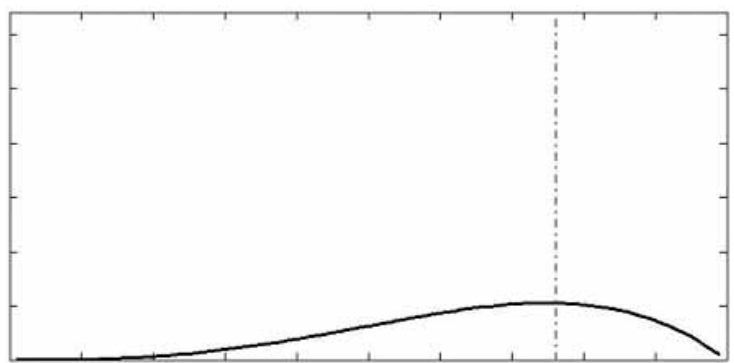

(b) - right-skewed Beta

Fig. 9. Shapes of the probability density functions assumed for material properties (a) and loads (b) variations.

The observed times-to-failure are ranked in ascending order from 1 to $n$ ( $n=20$, the number of runs within the stochastic simulation). For each of the ranks, the median ranks (MeR) are calculated and then transformed (TMeR), according to Eqs (16) and (17):

$$
\begin{aligned}
& M e R=\frac{\operatorname{Rank}-0.3}{n-0.4} \\
& T M e R=\ln \left\{\ln \left(\frac{1}{1-M e R}\right)\right\}
\end{aligned}
$$

where $\ln ($.$) stands for the natural logarithm, which is also applied to the time-to-failure figures.$

Considering the natural logarithms of the ranked time-to-failure figures as the independent variable $\mathrm{X}$ and the TMeR as the dependent variable $\mathrm{Y}$ in a regression analysis [6], Eq. (18) results:

$$
\ln (T T F)=\mu+\eta \cdot T M e R
$$

The slope $\eta$ of this straight line equation is the shape parameter of the Weibull probability distribution. Its statistical interpretation is given in Table 2 [7]:

Besides the shape parameter, the intercept $\mu$ is related to the characteristic life (Lc), equivalent to the time at which the $63 \mathrm{rd}$ percentile of the population involved in a stochastic process is expected to experience failure. From $\eta$ and 
Table 2

Statistical interpretation of the Weibull shape parameter

\begin{tabular}{cl}
\hline Shape Parameter Value & Interpretation \\
\hline$<1$ & $\begin{array}{l}\text { The failure rate decays with time. Usually, this happens in the beginning } \\
\text { of a system operation, where parts have to adapt to each other as to } \\
\text { function properly } \\
\text { The failure rate is a random constant because the system operates in a } \\
\text { steady state. The Weibull distribution assumes the form of one of its } \\
\text { special cases, the Exponential distribution } \\
\text { The failure rate increases with time. Usually this happens at the end of } \\
\text { the useful service life, known as "burn-out" phase }\end{array}$ \\
\end{tabular}

$\mu$, the characteristic life can be calculated as stated in Eq. (19):

$$
L c=e^{-\frac{\mu}{\eta}}
$$

For the case of the stochastic simulation data in Table 1, the straight line fit has a squared correlation coefficient of $85.07 \%$, a high value that supports the assumption of Weibull distributed failure probabilities. Furthermore, the slope and intercept equal 2.20 and -11.01 respectively, which yields a characteristic life of 148 load cycles. Indeed, inspection of Table 1 reveals that 15 in 20 (i.e., 75\%) of the population failed up to this characteristic life. The deviation from the theoretical percentile is equal to $19.04 \%$ and can be largely explained by the $14.93 \%(100.00 \%$ $-85.07 \%)$ lack of fit, besides the relatively small population size $(n=20)$.

Moreover, the 2.20 shape parameter indicates that the conservative environment set-up for the stochastic simulation resulted in a "burn-out" type of operation.

\section{Optimization}

Design optimization techniques consist of a mature tool for engineering [13]. For their successful application, an analysis model and/or databases are required. In the present case, the analysis model developed in Section 5 is employed to generate a database for the construction of response surfaces [6] to be used within an optimization procedure involving the foremost quantities of a coupled vibration-degradation phenomenon. The response surface approach is suitable for providing insight about the system to be optimized. At the same time, it is capable of reducing the cost for solving the optimization problem. This feature is particularly important for the procedures to be defined within this section because reliability measures, which call for the execution of whole stochastic simulations each time they need to be evaluated, are to be incorporated into the optimization formulation.

Actually, certain ranges around the original values of beam length (3.L), cross section width (b) and cross section height (h) will be explored as to maximize the characteristic life and minimize the shape parameter, in order to relieve the failure acceleration rate or "burn-out".

This way, two objective functions are defined, and will be treated by means of two separate optimization procedures. Both of them will account for constraints imposed over the weight of the beam (no greater than $10.00 \%$ above of the original value) and over its fundamental frequency for the intact condition (above $10.00 \mathrm{~Hz}$, which can be considered far enough from the excitation frequency of $5.00 \mathrm{~Hz}$ ).

Given the three design variables $\mathrm{L}, \mathrm{b}$ and $\mathrm{h}$, a central composite design is defined for them as in Table 3, aiming at the creation of quadratic response surface models. The yields are shown in Eqs (19) to (22), already considering only the $95.00 \%$ significant terms:

$$
\begin{aligned}
& L c=147.6292-24.3766 \cdot L+29.5686 \cdot h \\
& \eta=2.19899-0.225525 \cdot h \\
& \text { Weight }=1.716+0.3432 \cdot\{L+b+h+L \cdot b+L \cdot h+b \cdot h\} \\
& \omega_{n 1}=11.21865-5.17703 \cdot L+1.64838 \cdot L^{2}+0.86229 \cdot b^{2} \\
& +2.544234 \cdot h+0.86229 \cdot h^{2}-0.50968 \cdot L \cdot h
\end{aligned}
$$


Table 3

Central composite experimental design for response surface based optimization

\begin{tabular}{cccccccc}
\hline Run & $\mathrm{L}(\mathrm{m})$ & $\mathrm{b}(\mathrm{m})$ & $\mathrm{h}(\mathrm{m})$ & $\mathrm{Lc}$ & $\eta$ & Weight $(\mathrm{N})$ & Fundamental Frequency $-\omega_{n 1}(\mathrm{~Hz})$ \\
\hline 1 & 0.2700 & 0.0090 & 0.0180 & 145.00 & 2.22 & 1.250964 & 13.8761194 \\
2 & 0.2700 & 0.0090 & 0.0220 & 179.00 & 2.02 & 1.528956 & 16.9597015 \\
3 & 0.2700 & 0.0110 & 0.0180 & 175.00 & 2.04 & 1.528956 & 13.8761194 \\
4 & 0.2700 & 0.0110 & 0.0220 & 189.00 & 1.88 & 1.868724 & 16.9597015 \\
5 & 0.3300 & 0.0090 & 0.0180 & 152.00 & 2.11 & 1.528956 & 9.28897249 \\
6 & 0.3300 & 0.0090 & 0.0220 & 141.00 & 2.26 & 1.868724 & 11.3531886 \\
7 & 0.3300 & 0.0110 & 0.0180 & 126.00 & 2.33 & 1.868724 & 9.28897249 \\
8 & 0.3300 & 0.0110 & 0.0220 & 153.00 & 2.11 & 2.283996 & 11.3531886 \\
9 & 0.2495 & 0.0100 & 0.0200 & 160.00 & 2.07 & 1.427404 & 9.15148427 \\
10 & 0.3505 & 0.0100 & 0.0200 & 130.00 & 2.28 & 2.004596 & 12.4885075 \\
11 & 0.3000 & 0.0083 & 0.0200 & 150.00 & 2.16 & 1.427404 & 10.38851992 \\
12 & 0.3000 & 0.0117 & 0.0200 & 154.00 & 2.10 & 2.004596 & 14.5888157 \\
13 & 0.3000 & 0.0100 & 0.0166 & 122.00 & 2.37 & 1.427404 & 11.2100000 \\
14 & 0.3000 & 0.0100 & 0.0234 & 204.00 & 1.71 & 2.004596 & 11.2100000 \\
15 & 0.3000 & 0.0100 & 0.0200 & 148.00 & 2.20 & 1.716000 & \\
16 & 0.3000 & 0.0100 & 0.0200 & 148.00 & 2.20 & 1.716000 &
\end{tabular}

Table 4

Design optimization results, from response surface estimates

\begin{tabular}{clcccccr}
\hline Design Problem & $\mathrm{L}(\mathrm{m})$ & $\mathrm{b}(\mathrm{m})$ & $\mathrm{h}(\mathrm{m})$ & $\mathrm{Lc}$ & $\eta$ & Weight $(\mathrm{N})$ & Fundamental Frequency $-\omega_{n 1}(\mathrm{~Hz})$ \\
\hline 1 & 0.249546 & 0.01 & 0.023364 & $+1.94 \%$ & $-0.035 \%$ & $-0.08 \%$ & $+2.27 \%$ \\
2 & 0.3 & 0.01 & 0.023364 & $+0.07 \%$ & $+0.035 \%$ & $+0.08 \%$ & $+0.08 \%$ \\
\hline
\end{tabular}

An inspection of the signals and relative values of the coefficients demonstrate that these response surface models are consistent from the physical viewpoint. Besides, the less accurate of them has a squared multiple correlation coefficient of $78.5 \%$, and the best one has above $99.00 \%$ of explained variance. Therefore, their predictive capabilities can be trusted for the sake of the two proposed optimization procedures.

For both of the optimization procedures, the resulting percent variations in the responses of interest are shown in Table 4, along with the corresponding optimum design variables values. These solutions were obtained by means of the Newton method, whose employment is facilitated by the availability of analytical response surface models representing the objective and constraint functions, from which inexpensive closed form Hessian matrices can be easily deployed.

All these optima are feasible, in spite of the stringent constraints imposed in both formulations. In the first case, the characteristic life is improved in $1.94 \%$. This is a very significant improvement obtained for a response that depends upon a large number of parameters and is evaluated in the midst of a stochastic simulation geared towards the "burn-out" regime. Under more realistic operational conditions, which are far less adverse than those considered in this scenario, the improvement in life would be increased as well, leading to substantial economy at the operation of aircraft designed to undergo tens of thousands flights.

The reduction of the failure rate acceleration factor (Weibull shape parameter $\eta$ ) resulted to be much less successful, which can be credited to severity of the "burn-out" setup created in the framework of the stochastic simulation, against which it is difficult to obtain an improved design. Problems related to the optimization algorithm itself are ruled out because its influence is rather limited in this case, where quadratic and well behaved response surface models are responsible for representing the design space.

It should be noted that with the power of optimization techniques and the flexibility of the response surface method, several optimization procedures can be formulated and solved at low cost, enabling the achievement of design gains with respect to many of the criteria assessed by means of the working model developed in Section 5 .

\section{Discussion}

This paper has highlighted the importance of the damage tolerant design philosophy to ensure structural safety for aircraft operation, and proposed a means of improving life estimates for damaged (cracked) components that undergo dynamic loading. 
A coupled vibration-degradation problem has been stated and a working model for its solution has been proposed. This model could be used to obtain insight about the vibration-degradation phenomenon, as well as to perform reliability based optimization procedures.

The aim of this section is to present a brief outline of future work possibilities. Among the many existing possibilities, future research effort shall concentrate on exploring the behaviour of the coupled vibration-degradation problem encompassing more aspects of its dynamics, such as:

- The example shown in this work does not specify any damping for the cantilever beam. Is it possible to determine if there is any relation between the system structural damping and the crack propagation?

- The excitation frequency of $5.00 \mathrm{~Hz}$ is lower than the fundamental vibrating frequency, meaning that the motion of the beam is primarily driven by the (first modal) stiffness. What would happen in higher frequencies, with the influence of higher order vibrating modes?

- The effect of non-linear modelling for the vibratory motion shall be investigated.

- Besides the variation in the load magnitude, a comprehensive random vibrations study could be implemented within the stochastic simulation framework.

Furthermore, instead of assuming a place for the crack onset, multi site crack growth shall be performed. This requires the realization of case studies involving more realistic structures, and a stochastic framework could be also used while considering the multiple crack locations.

Finally, from the reliability studies viewpoint, one could consider the application of techniques devoted to the analysis of censored data, as to contemplate those stochastic simulation outcomes by which a specific combination of random parameters prevents the crack to grow, and there is no time to failure data available. This encompasses the replacement of the Median Ranks Weibull analysis by Maximum Likelihood methods.

\section{Conclusion}

This paper has highlighted the importance of the damage tolerant design philosophy to ensure structural safety for aircraft operation, and proposed a means of improving life estimates for damaged (cracked) components that undergo dynamic loading.

A coupled vibration-degradation problem has been stated and a working model for its solution has been proposed. This model could be used to obtain insight about the vibration-degradation phenomenon, as well as to perform reliability based optimization procedures.

The results obtained by means of an illustrative test case stress the importance of the assumptions made upon the basic models aimed at representing the behaviour of a set of non trivial phenomena, besides choosing parameter values that often require intense experimental work.

\section{Acknowledgements}

The author wishes to express his thanks to:

- EMBRAER, Empresa Brasileira de Aeronáutica S.A., for the support on research and development initiatives that resulted in this paper;

- The organizing committee of the XI DINAME, for their kind assistance during all the preparation, presentation and publication process;

- The SAV reviewers, whose suggestions brought significant contributions to the improvement of the original manuscript.

\section{Responsibility notice}

The author is the only responsible for the printed material included in this paper. 


\section{References}

[1] G.R. Buchanan, Finite Element Analysis, Schaum's Outlines, McGraw-Hill, New York, USA, 1994.

[2] K.C. Chou, G.C. Cox and A.M. Lockwood, Crack Growth Life Model for Fatigue Susceptible Structural Components in Aging Aircraft, Structural Engineering and Mechanics 17(1) (2004), 29-50.

[3] R.R. Craig, Structural Dynamics: An Introduction to Computer Methods, John Wiley \& Sons, New York, USA, 1981.

[4] N.E. Dowling, Mechanical Behavior of Materials: Engineering Methods for Deformation, Fracture and Fatigue, Prentice Hall, Portland, USA, 1998.

[5] M. Evans, N. Hastings and B. Peacok, Statistical Distributions, Wiley Interscience, Hoboken, USA, 2000.

[6] A.I. Khury and J.A. Cornell, Response Surfaces: Designs and Analysis, Marcel Drekker Inc., New York, USA, 1987.

[7] J.R.B. Lafraia, Reliability, Maintenability and Availability Handbook (in Portuguese), Qualitymark, Rio de Janeiro, Brazil, 2000.

[8] M. Lalanne, P. Berthier and J. Der Hagopian, Mechanical Vibrations for Engineers, John Wiley \& Sons, New York, USA, 1984.

[9] B.L. Nelson, Stochastic Modelling, Analysis \& Simulation, Dover Publications Inc., Mineola, USA, 1995.

[10] S.S. Rao, Reliability Based Design, MacGraw-Hill Inc., New York, USA, 1992.

[11] S.M. Ross, Applied Probability Models with Optimization Applications, Dover Publications Inc., New York, USA, 1970.

[12] K. Sobczyk and J. Trebicki, Random Vibration of Fatigue Degrading Systems, Proceedings of the 8th American Society of Civil Engineers Specialty Conference on Probabilistic Mechanics and Structural Reliability, Notre Dame, USA, 2000.

[13] G.N. Vanderplaats, Industrial Applications of Optimization, Proceedings of the 10th AIAA/ISSMO Multidisciplinary Analysis and Optimization Conference, Albany, USA, 2004. 

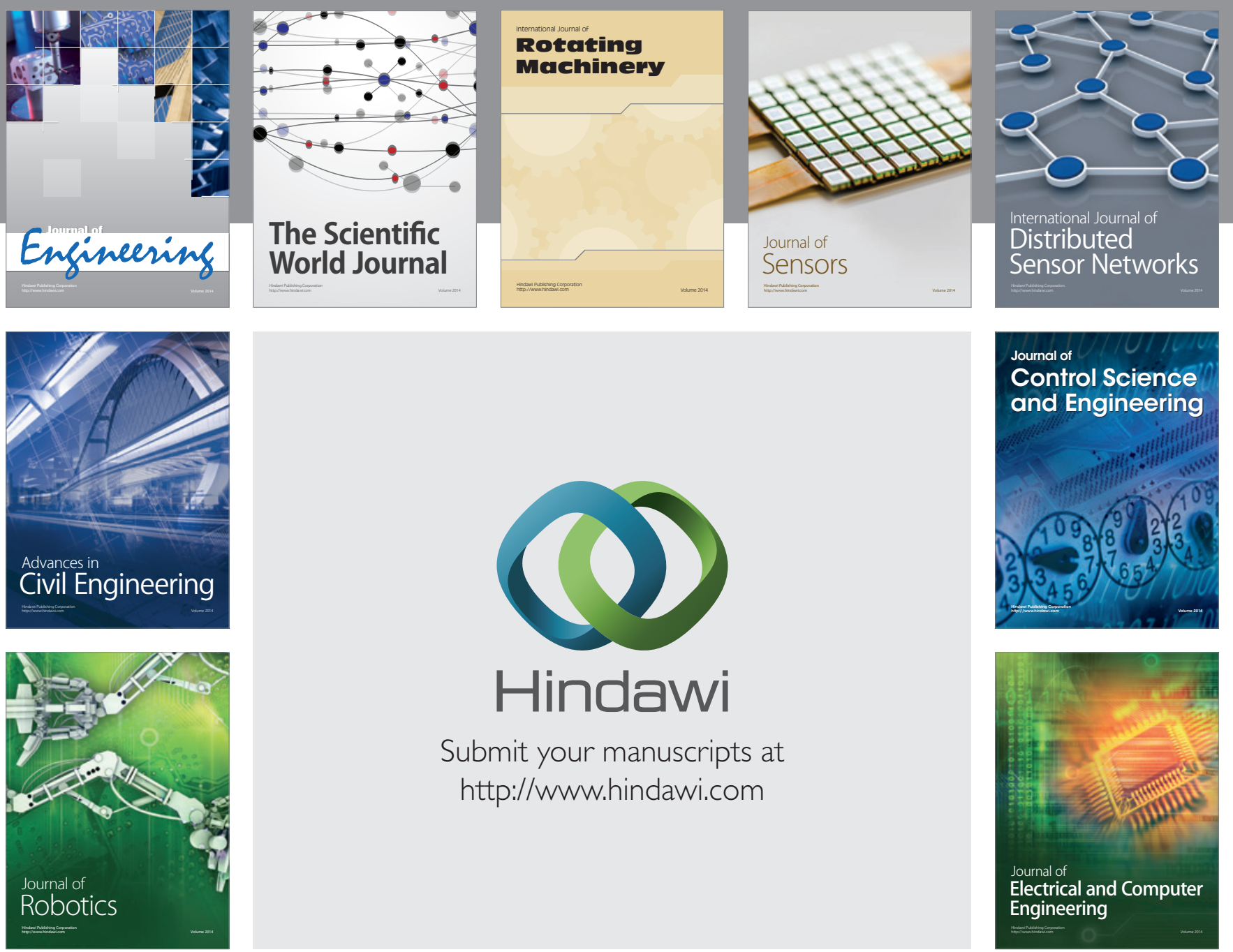

Submit your manuscripts at

http://www.hindawi.com
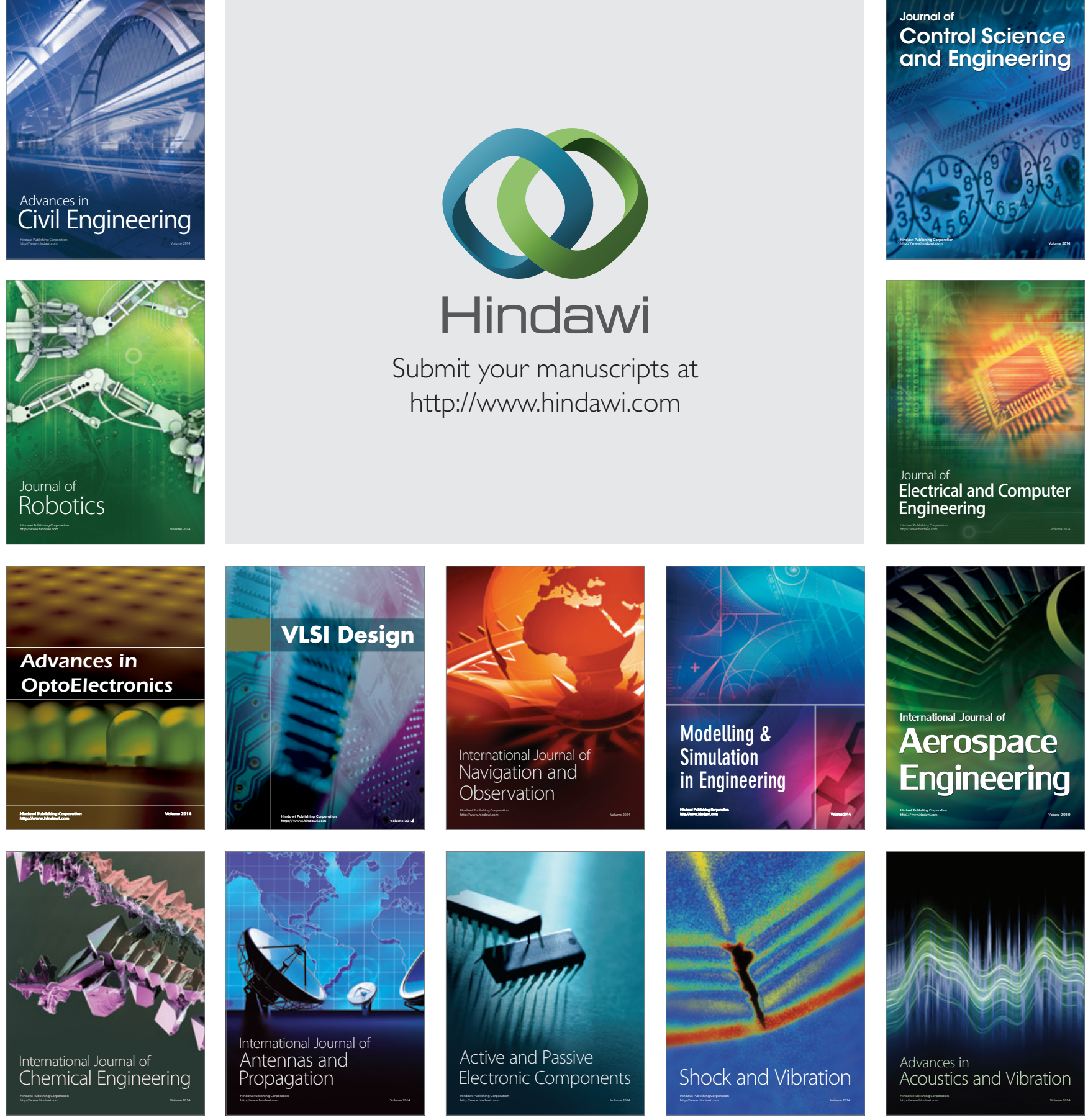